\title{
LA SFIDA DELLA DIDATTICA A DISTANZA IN UNA UNIVERSITÀ SUDANESE: LA VALUTAZIONE DEGLI STUDENTI E I RISULTATI NEI CORSI DI LINGUA ITALIANA
}

\author{
Jorge Naranjo, Ilaria Molineri ${ }^{1}$
}

\section{INTRODUZIONE}

Il 15 marzo 2020 tutte le università sudanesi sono state costrette a chiudere a causa dell'epidemia di COVID 19. Il 21 giugno 2020, il Ministero dell'Educazione Superiore e della Ricerca Scientifica della Repubblica del Sudan ha incoraggiato gli atenei a sondare la possibilità di riprendere le attività accademiche attraverso piattaforme per lo studio da remoto, nonostante le molteplici sfide che una tale iniziativa avrebbe comportato, a causa di interruzioni di corrente, accesso limitato o irregolare a Internet, scarsa preparazione del corpo docente, competenze digitali molto limitate tra gli studenti.

La pandemia è scoppiata quando la nazione si trovava in un periodo di transizione, in cui il normale corso delle attività accademiche era stato interrotto previamente durante alcuni momenti della rivoluzione popolare (dicembre 2018 - agosto 2019) che ha portato alla fine di trenta anni di regime militare e islamista.

Il 17 agosto 2020, il Comboni College of Science and Technology (CCST), un college universitario privato situato a Khartoum, ha abbracciato con entusiasmo e ottimismo l'enorme sfida, forte di un progetto educativo visionario e di un meticoloso lavoro di costruzione e internalizzazione del campus a partire dal 2001, anno della sua fondazione, evoluzione naturale del liceo aperto nel 1929 dai Missionari Comboniani, il Comboni College Khartoum (CCK). L'offerta didattica del CCST comprende tre lauree in Scienze Informatiche: Informatica, Tecnologia dell'Informazione (IT) applicata all'Amministrazione e alla Contabilità, Lingua e Letteratura Inglese (con scelta di una seconda lingua tra italiano e spagnolo) e Scienze dell'Educazione e Religione Cristiana, a beneficio di circa 700 iscritti.

Il secondo semestre dell'anno accademico 2019-2020 è iniziato proponendo corsi ibridi, composti da lezioni teoriche a distanza attraverso il Learning Management System (LMS) Moodle e seminari in presenza, in piccoli gruppi, presso i laboratori di informatica.

L'obiettivo di questa ricerca è valutare l'esperienza formativa degli studenti che hanno utilizzato Moodle, anche attraverso l'analisi dettagliata dei risultati ottenuti nei corsi di lingua italiana, afferenti al programma di Laurea in Lingua e Letteratura Inglese, e ricavare raccomandazioni sull'eventuale, futura integrazione delle lezioni in remoto nell'offerta didattica dell'ateneo.

Su un piano più generale, si intende fornire uno spunto rispetto alla possibilità di introdurre la didattica a distanza in contesti simili a quello di riferimento, in cui la connettività, la fornitura elettrica e le abilità digitali sono molto limitate e non solo una pandemia, ma la situazione politica e sociale determina spesso l'interruzione delle attività scolastiche di ogni ordine e grado o limita fortemente l'accesso alle stesse da parte della popolazione.

\footnotetext{
${ }^{1}$ Comboni College of Science and Technology (CCST), Khartum, Sudan.
} 


\section{CORNICE TEORICA}

L'obiettivo principale della ricerca è dunque quello di valutare l'esperienza formativa degli studenti universitari che hanno utilizzato la piattaforma Moodle, dopo circa un mese di somministrazione di corsi online tra agosto e settembre del 2020 in un'istituzione di educazione superiore in Sudan.

A tal fine, è stato necessario verificare le competenze digitali degli studenti prima dell'introduzione del LMS, come anche le competenze linguistiche e il livello di inglese, poiché il Sudan è un paese di lingua araba, mentre la lingua di insegnamento e quindi di lavoro della piattaforma è l'inglese.

L'utente/discente faticherà a interagire efficacemente in una riunione online fino a che non avrà raggiunto $i$ livelli $\mathrm{B}$ di competenza linguistica; sarà in grado di partecipare a lezioni virtuali a un livello A2 solo se attentamente guidato e potrà forse comunicare in maniera molto semplice a livello A1, pubblicando commenti o utilizzando la chat, in un ambito di "internet cafe" (North, Goodier, Piccardo, 2018)².

Ne è conseguita l'elaborazione di linee guida per l'integrazione della didattica a distanza attraverso il LMS nell'offerta formativa di un'istituzione universitaria, in un contesto simile a quello sudanese, condizionato da frequenti interruzioni della fornitura elettrica, difficoltà di accesso alla rete Internet e caratterizzato dalla dicotomia tra lingua d'uso comune e lingua accademica.

\subsection{Formazione online e TIC nelle università sudanesi}

Mentre tutte le università sudanesi dispongono di laboratori informatici e connessione Internet, la maggior parte delle scuole e licei pubblici del paese, fino a qualche anno fa, non era equipaggiata in tal senso (Hamdy, 2007). Ancora oggi, quasi tutti gli studenti che accedono alla formazione superiore provenendo dalle scuole pubbliche non hanno mai utilizzato un computer. Durante il primo anno, i curricula universitari includono un corso di alfabetizzazione informatica.

Per quanto riguarda invece le università telematiche, in Sudan sono presenti la Open University of Sudan, che utilizza Moodle come LMS, e la Arab Open University. In generale, gli studenti iscritti sono adulti e le altre università non prendono in considerazione l'opzione di reclutarli come docenti dopo la laurea, considerandoli come laureati di livello più basso in relazione a quello delle università presenziali.

Nel 2009, Omer Hassan Abdelrahman, professore associato presso il dipartimento di Biblioteconomia e Scienze dell'Informazione dell'università di Khartoum, concluse un'indagine sulla formazione online in Sudan con una lista di problemi che rimangono ancora estremamente attuali dodici anni più tardi:

Scarse infrastrutture TIC in termini di hardware e dotazioni; mancanza di programmi di alfabetizzazione informatica per studenti e insegnanti; mancanza di forza lavoro con competenze informatiche nel settore dell'educazione; lentezza nell'introdurre nei curriculum scolastici nazionali corsi di scienze informatiche; scarsa consapevolezza e impegno da parte della classe dirigente nel riconoscere l'importanza dell'introduzione delle TIC nei

\footnotetext{
2 Tutte le citazioni sono tratte da fonti anglofone e sono state tradotte da Ilaria Molineri.
} 
programmi scolastici; mancanza di materiali didattici sia per l'istruzione inferiore che per quella superiore (Hassan Abdelrahman, 2010: 308).

Una ricerca successiva di Negla Ahmed Albasheer (2014) sul livello di accettazione e l'utilizzo della tecnologia da parte degli insegnanti presso le università pubbliche di Khartoum «ha rivelato che le TIC sono largamente impiegate dal corpo docente. Un numero relativamente basso però le sfrutta per l'insegnamento» (ibidem: I). L'indagine ha anche evidenziato come uno dei fattori che ne ostacolano un impiego più diffuso è «la preponderanza su internet di contenuti in inglese», mentre la prima lingua del paese è l'arabo (Ahmed Albasheer Osman, 2014: 164). Un altro dato interessante per il nostro studio è che mentre l'informatica è stata introdotta nelle università sudanesi agli inizi degli anni Novanta, i servizi per la connessione a Internet sono arrivati più tardi, verso la fine del ventesimo secolo. Perciò, mentre circa il 32\% degli intervistati aveva avuto circa dieci anni di esperienza con i computer, solo il $16 \%$ ne aveva avuto una simile con Internet (Ahmed Albasheer Osman, 2014: 187).

\subsection{Internet in Sudan}

A gennaio 2020, la connessione dati mobili raggiungeva 32,83 milioni di persone, circa il 76\% della popolazione sudanese. Secondo Kemp (2020) gli utenti Internet erano 13,38 milioni, vale a dire il 31\% della popolazione. Queste statistiche sono simili a quelle presentate dal sito Internet World Stats, che riporta come al 30 settembre 2020 il Sudan avesse 13.124.100 di utenti Internet e un tasso di penetrazione del 29,9\%.

Il Sudan si classifica al centosessantaquattresimo posto su 207 paesi in termini di velocità di connessione, con una media di $1.94 \mathrm{Mbps}$ e un tempo di download per 5GB di 5 ore e 53 minuti (Hananto, 2019). La relazione di Kemp invece riporta una velocità media di connessione Internet mobile di 8.60 Mbps. Questa velocità è diminuita del 19\% lo scorso anno. La velocità media di connessione via cavo è di $6.81 \mathrm{Mbps}$ (Kemp, 2020).

La Intelligence Unit della rivista Economist pubblica l'Inclusive Internet Index, che esamina il costo dell'accesso alla rete rispetto al reddito e il livello di competizione tra fornitori sul mercato. Nell'edizione del 2020, il Sudan si è classificato settantacinquesimo su 100 paesi alla voce convenienza (affordability). Quanto alla disponibilità (availability), la stessa fonte considera «la qualità e la gamma di infrastrutture disponibili, fondamentali per l'accesso, nonché i livelli di utilizzo». In questo caso, il Sudan si è piazzato novantacinquesimo su 100 paesi. Infine, con riferimento alla predisposizione (readiness), definita come «capacità di accesso a internet, comprese abilità, competenze interculturali e policy a supporto», il Sudan ha raggiunto il novantasettesimo posto su 100 paesi (The Economist, 2020).

Il 62,3\% degli utenti accede al web attraverso il telefono cellulare e il 37,2\% attraverso computer portatili o fissi. Quest'ultimo dato è cresciuto del 97\% tra dicembre $2018 \mathrm{e}$ dicembre 2019. Il tasso di crescita annuale è del 2,4\%, vale a dire di oltre 316.000 nuovi utenti all'anno (Kemp, 2020).

Tra i siti più visitati troviamo i conosciuti Google, Youtube, Facebook, Yahoo, Wikipedia, Live e Blogspot. L'ottavo è un sito web locale, Sudanakhbar.com (Kemp, 2020: 24). Gli utenti Facebook in Sudan erano 1.300.000 a settembre 2020 (Internet World Stats, 2020). 1,30 milioni $(3.0 \%)$ di sudanesi sono attivi sui social media. L'applicazione di messaggistica più diffusa in Sudan è Whats $A p p$. Vale la pena menzionare che le ricerche effettuate su Youtube si riferiscono in gran parte a film e canzoni (Kemp, 2020). Il traffico dati generato da dispositivi mobili è per il 93,1\% proveniente dal sistema operativo Android e per il 3,2\% da quello Apple (Kemp, 2020). 


\subsection{La risposta al COVID-19}

Come sopra accennato, il 14 marzo 2020 il Consiglio dei ministri sudanese ha deciso la chiusura delle scuole e università a causa dell'epidemia di corona virus. Tre mesi più tardi, il 21 giugno 2020, il Ministro dell'Educazione Superiore e della Ricerca Scientifica, Prof. Intisar Saghayroun, ha esortato le università pubbliche e private ad avviare programmi di didattica a distanza.

In Sudan ci sono 53 università e 81 college universitari. Poche istituzioni hanno saputo trasformare quella sfida nell'opportunità di avviare programmi di studio da remoto attraverso Learning Management Systems (LMS). Nel 2020, il sito ufficiale di Moodle registrava otto atenei sudanesi attivi sulla piattaforma (Moodle, 2020).

Il Ministero ha selezionato sei università affinché il personale fosse formato e agli studenti fosse garantito accesso gratuito alle reti mobili (Sawahel, 2020). Nonostante questi sforzi, la maggior parte, tra cui la prestigiosa università di Khartoum, hanno ritenuto di non essere pronte a un tale passo, a causa di «infrastrutture carenti, instabilità delle forniture elettriche, disponibilità di Internet e problemi finanziari, con la maggior parte degli studenti privi di accesso a un $p c$ e con difficoltà a coprire i costi per la connessione» (Gamal Abdo, citato da Sawahel, 2020). Secondo il Professor Gamal Abdo, «il sondaggio effettuato tra insegnanti e studenti dell'università di Khartoum evidenziava una scarsa capacità di introdurre la didattica a distanza in termini di materiali didattici e dotazioni, formazione del corpo docente e non e scarse risorse finanziarie» (Gamal Abdo, citato da Sawahel, 2020). Vi era inoltre la sfida di dover convincere la popolazione universitaria, ma non solo, della necessità di intraprendere un cambiamento di quel tipo, poiché «non si introduceva solo una nuova tecnologia per lo studio a distanza, ma un nuovo modo di pensare all'apprendimento, una nuova cultura della formazione» (Eltahir, citato da Sawahel, 2020).

\subsection{Moodle}

La piattaforma utilizzata dal Comboni College of Science and Technology e da gran parte delle università sudanesi, è stata (ed è tuttora) Moodle, un LMS open source tra i più quotati (Graf, List, 2005; Cavus, Zabaldi, 2014). Introdotta negli anni precedenti, era sfruttata principalmente come repository di materiale didattico.

Moodle, originariamente acronimo di ModularObject-Oriented Dynamic Learning Environment (ambiente per l'apprendimento modulare dinamico orientato a oggetti), si fonda su basi di pedagogia sociale costruttivista, cioè «[sul]l'approccio collaborativo all'apprendimento, secondo le teorie di Jerome Bruner, Lev Vygotsky e Jean Piaget» (Crosslin, 2009: 505), che «consente alla formazione online di superare il tradizionale ambito educativo, basato sul nozionismo e la memorizzazione, per giungere alla costruzione di reti sociali» (Crosslin, 2006: 505).

La relazione dell'Unesco sul sistema educativo sudanese menziona «il ricorso alla memorizzazione come metodo primario per l'acquisizione, anche per materie quali matematica e scienze, che richiedono competenze e abilità analitiche» (Watanebe, 2018: 99). Il cambio di prospettiva derivante dall'introduzione della piattaforma Moodle appare perciò significativo.

Affinché l'esperienza con il LMS sia positiva, uno degli aspetti fondamentali è il livello di soddisfazione degli utenti, che a sua volta dipende da fattori che Sun et al. hanno categorizzato «in sei dimensioni: studente, docente, corso, tecnologia, disegno di sistema e ambiente» (Sun, Tsai, Finger, Chen, Yeh, 2008: 1184). 


\subsection{Moodle al CCST}

Dopo l'annuncio del Mistero dell'Educazione Superiore, l'amministrazione del CCST si è riunita e ha deliberato di predisporre l'avvio del secondo semestre con un'offerta formativa di corsi ibridi, proponendo contenuti teorici attraverso la piattaforma e lezioni pratiche in piccole classi di quindici persone nei laboratori informatici per i programmi di laurea in Scienze Informatiche. Per gli studenti di Lingua e Letteratura Inglese sono stati predisposti classi di conversazione e seminari, in aggiunta ai contenuti erogati a distanza.

Durante il mese di luglio 2020, i dipartimenti hanno organizzato workshop per gruppi di una decina di docenti sui seguenti temi: il cambiamento di mentalità necessario all'implementazione della didattica sulla piattaforma, all'interno della quale i docenti cessano di essere dispensatori di informazioni, diventando gestori dell'esperienza formativa degli studenti o della comunità di apprendimento; l'utilizzo di Internet da parte della gioventù sudanese; le motivazioni per l'introduzione di Moodle e formazione tecnica e pedagogica per favorire l'efficacia.

In considerazione delle frequenti interruzioni di forniture elettriche che affliggevano il paese all'epoca, la scarsa velocità di download e il limitato accesso a Internet da parte degli studenti, si è deciso di non ricorrere a video-lezioni o video-conferenze (sincrone o asincrone), ma di caricare i contenuti didattici su file PDF scaricabili e di promuovere l'interazione attraverso gli strumenti di Moodle quali chat room sincrone e forum asincroni.

Durante la prima settimana di un secondo semestre certamente particolare, dal 17 al 22 agosto, presso i laboratori informatici del college, gli studenti hanno partecipato a workshop di addestramento all'uso di Moodle, su cui la maggior parte dei docenti aveva già caricato il materiale didattico.

Il 14 settembre, le lezioni delle materie più complesse sono riprese in presenza e il 21 settembre la totalità degli studenti è tornata in classe; poiché il docente di italiano non era in Sudan, le lezioni di lingua sono invece continuate in modalità ibrida fino alla fine del semestre.

\subsection{I corsi di italiano al CCST}

I corsi d'italiano al Comboni College (CCK) sono incominciati con la sua creazione nel 1929, indirizzati allora ai figli della numerosa comunità italiana residente in Sudan. Nel 2002 il Ministero delle Università e la Ricerca Scientifica sudanese ha autorizzato i programmi di educazione continua proposti dalla appena costituita sezione universitaria, il CCST. Nel 2011, lo stesso Ministero ha approvato una Laurea di Lingua e Letteratura Inglese, dove l'italiano era inserita come seconda lingua. Nel 2015, il CCST è diventato centro di esami CELI, mentre nel 2016, con l'appoggio dell'ambasciata italiana a Khartoum, è nato il Comboni Italian Center all'interno del CCST, un "ambiente italiano" che facilita l'apprendimento della lingua tanto per gli studenti del corso di laurea come per quelli dei corsi di formazione continua.

Lo studio della lingua italiana quindi forma parte del sillabo della laurea in Lingua e Letteratura Inglese; al secondo e terzo anno, gli studenti scelgono tra italiano e spagnolo come seconda lingua per un totale di quattro semestri, con l'obiettivo di raggiungere il livello A2 del Quadro Comune Europeo di Riferimento per le Lingue.

Il 17 agosto 2020, i programmi di italiano sono iniziati interamente a distanza, con sessioni in chat sincrona organizzate settimanalmente e frequenti messaggi su Moodle per supportare e verificare se e come gli studenti accedessero ai contenuti caricati, e per orientarli all'uso interattivo del LMS, all'organizzazione del tempo di studio e 
all'integrazione del materiale online con il libro di testo, già utilizzato nel semestre precedente, in presenza, con un altro insegnante. Non erano ancora attivi gruppi $W$ hatsapp e, come si è poi verificato successivamente, quasi tutti erano scarsamente abituati a utilizzare i loro account di posta elettronica.

A metà settembre, con il ritorno in classe, il programma di italiano è stato ristrutturato come segue: due ore a settimana in videoconferenza con l'insegnante madrelingua collegato con la classe al college, supportato dall'assistente locale, e due ore a settimana di laboratori pratici condotti da quest'ultimo in autonomia, precedentemente preparati con il docente, a integrazione di quanto svolto e al materiale disponibile su Moodle, sempre monitorato e commentato. L'assistente, formato nel corso degli anni, ha continuato la formazione a distanza, parallelamente al lavoro di classe.

I contenuti disponibili su Moodle includevano: brevi video tutorial (1-2 minuti ciascuno) a illustrare dove trovare il materiale didattico, con spiegazione a voce in inglese e schermo condiviso dei passaggi da compiere per completare le attività (quiz, forum, wiki di Moodle); documentazione generale (guida del corso e guida orientativa su studio online e materiale extra, criteri di valutazione); programma settimanale per ciascuna unità del libro di testo, con istruzioni, esercizi e riferimenti grammaticali; video didattici incorporati di circa 5-7 minuti con spiegazione dei principali punti grammaticali o attività di vocabolario, realizzati dal docente o condivisi tra quelli disponibili su YouTube, anche per favorire l'esposizione a tecniche d'insegnamento e accenti differenti; attività di autoapprendimento, tra cui quiž e forum per lo sviluppo delle abilità di scrittura e file audio o testo per addestrare abilità di ascolto e lettura. Per ogni attività era specificato un tempo stimato da dedicarvi, per un totale di 4 ore a settimana, come previsto dal sillabo.

Quanto alla valutazione, si è mantenuto l'impianto generale approvato dal Senato Accademico di due esami parziali scritti in itinere ( $40 \%$ del voto) e un esame finale scritto (60\% del voto) in presenza. Ai punteggi degli esami parziali si sono sommati anche quelli della valutazione continua, che ha considerato la partecipazione attiva ai laboratori di conversazione in presenza e il monitoraggio delle attività di autoapprendimento su Moodle. Per esempio: si è tenuto conto del fatto che il quiz fosse stato eseguito (o meno) e non della percentuale di risposte corrette, così come dell'esecuzione (o meno) delle esercitazioni in classe e del livello di impegno. Rispetto alla presenza di almeno il $75 \%$ delle lezioni, requisito fondamentale per l'ammissione agli esami finali in semestri normali, si è deciso per una maggiore flessibilità, considerato che lo stop per la pandemia è coinciso con la normale pausa estiva tra semestri e, nel 2020, la fine delle vacanze di Ramadan, quando cioè gli studenti si erano allontanati da Khartoum per raggiungere le famiglie; alla ripresa delle lezioni, non è stato possibile per molti tornare immediatamente in città, sia per la scarsa disponibilità di mezzi di trasporto, dovuta a forniture di gasolio insufficienti in tutto il Sudan, sia per motivi economici.

\section{Progetto di Ricerca E MEtodologia}

\subsection{Temi della ricerca, obiettivi e strategia}

L'obiettivo della ricerca - valutare l'esperienza formativa degli studenti del CCST che hanno utilizzato Moodle - può tradursi in forma di domande principali, come segue:

1) Come è stata l'esperienza formativa degli studenti attraverso il LMS?

2) Come si può rendere maggiormente efficace l'utilizzo della piattaforma, date le specificità del contesto? 
La prima ha carattere descrittivo, mentre la seconda investigativo; si può quindi conferire alla ricerca un carattere sia descrittivo che investigativo.

Le sotto-domande sono dettagliate come segue:

a) Le competenze digitali pregresse degli studenti hanno influito sull'utilizzo di Moodle?

b) Il livello di competenza dell'inglese degli studenti ha avuto un impatto nell'accesso alla piattaforma?

c) Il gruppo di studenti che l'ha utilizzata per tutto il secondo semestre ha raggiunto risultati inferiori a quelli del primo semestre in presenza?

Considerando l'obiettivo, il fatto che non vi sia controllo sugli eventi comportamentali e che le domande si concentrano su eventi contemporanei, la strategia di ricerca più conveniente è la somministrazione di un questionario (Yin, 2002). Quanto alla valutazione quantitativa dei risultati (sotto-domanda c), lo studio propone il confronto tra i voti del primo semestre dell'anno accademico 2019-2020, svoltosi interamente in presenza, e il secondo, come descritto nella sezione 2.6. Vale la pena ribadire che si tratta degli stessi studenti, che hanno lavorato con lo stesso sillabo e libro di testo, ma con due insegnanti diversi e, ovviamente, con la componente online in aggiunta.

\subsection{Progetto di ricerca}

Come principale strumento di indagine si è scelto un questionario anonimo con domande articolate in tre parti. La prima mirava a raccogliere $i$ dati demografici e le informazioni circa il background linguistico degli studenti, le possibilità che essi avevano di collegarsi a internet e le loro abitudini al riguardo, prima di doversi cimentare con la piattaforma Moodle.

In questo modo si proporzionano quindi le informazioni per rispondere alle prime due sotto-domande.

La seconda parte del questionario si concentrava sulla valutazione dei workshop di orientamento e formazione sull'uso di Moodle.

Per entrambe le prime due parti le risposte erano a scelta multipla. Infine, la terza parte del questionario si proponeva di rispondere alle due domande principali, che esplicitano l'obiettivo primario della ricerca; le risposte sono state classificate in accordo con le sei dimensioni definite da Sun et al. (2008: 1184): «studente, insegnante, corso, tecnologia, sistema e ambiente».

Per quanto riguarda la terza sotto-domanda, lo studio descrive l'esperienza formativa degli studenti di italiano che hanno utilizzato Moodle per tutta la durata del corso e compara i voti del primo e del secondo semestre attraverso l'applicazione di test per dati appaiati. Poiché i campioni di studenti di italiano sono costituiti da meno di 30 individui, è stato scelto il test di Shapiro-Wilk per la verifica della normalità.

\subsection{Protocollo per la raccolta dei dati}

Il questionario è stato distribuito tra il 29 settembre e il 3 ottobre 2020, quando quasi tutti gli studenti avevano fatto ritorno in classe. Per non discriminare coloro con competenze informatiche inferiori, è stato stampato su carta e somministrato in classe.

I risultati delle risposte a scelta multipla sono stati trasferiti su un file Excel. Le risposte aperte sono state riportate su un file Word. 
Per l'analisi dell'esperienza formativa degli studenti di italiano, lo studio si riferisce al diario del docente, mentre è stato utilizzato il software SPSS Statistics (v. 21) per processare i dati.

\subsection{Popolazione studentesca, campioni e raccolta dati}

Il numero totale degli iscritti ad agosto 2020, all'inizio del secondo semestre dell'anno accademico 2019-2020, era di 677. I 77 studenti della Laurea in Scienze dell'Educazione e la Religione Cristiana non sono stati inclusi nello studio, poiché non hanno usufruito della piattaforma. Trattandosi infatti di piccoli gruppi di circa 20 persone, è stato possibile riprendere subito le lezioni in presenza, rispettando il distanziamento sociale.

Il questionario ha dunque riguardato gli studenti dei corsi di laurea in Informatica ( $B S c$ in Computer Science: CS), Lingua e Letteratura Inglese (BA in English Language and Literature: EN) e quelli dei diplomi universitari in IT per la Contabilità (Diploma in Information Technology-Accounting: ITA e Management. ITM)

Tabella 1. Numero degli intervistati

\begin{tabular}{lccccc}
\hline & CS & EN & ITM & ITA & Totale \\
\hline No. degli intervistati & 64 & 186 & 79 & 77 & 406 \\
\hline Totale studenti & 101 & 275 & 106 & 118 & 600 \\
\hline$\%$ & 63,37 & 67,64 & 74,53 & 65,25 & 67,67 \\
\hline
\end{tabular}

\section{RisulTATi}

\subsection{Retroterra linguistico e utilizzo di internet}

Prima di addentrarsi nella valutazione dell'esperienza formativa, il questionario si focalizzava sulla raccolta dei dati rispetto a caratteristiche degli studenti, retroterra linguistico e abitudini digitali (Tabella 2).

Tabella 2. Retroterra linguistico e nażionalità

\begin{tabular}{|c|c|c|c|c|c|c|c|c|c|c|}
\hline & & \multicolumn{2}{|c|}{ CS } & \multicolumn{2}{|c|}{ EN } & \multicolumn{2}{|c|}{ ITA } & \multicolumn{2}{|c|}{ ITM } & \multirow[b]{2}{*}{ Totale } \\
\hline & & No. & $\%$ & No. & $\%$ & No. & $\%$ & No. & $\%$ & \\
\hline \multirow{2}{*}{$\begin{array}{l}\text { Scuola primaria } \\
\text { in }\end{array}$} & arabo & 48 & 76,2 & 155 & 83,3 & 34 & 43,6 & 38 & 50 & 275 \\
\hline & inglese & 15 & 23,8 & 31 & 16,7 & 42 & 56,3 & 38 & 50 & 126 \\
\hline \multirow{2}{*}{ Secondaria in } & arabo & 47 & 75,8 & 154 & 85,6 & 29 & 48,2 & 36 & 48 & 266 \\
\hline & inglese & 15 & 24,2 & 26 & 14,4 & 47 & 61,8 & 39 & 52 & 127 \\
\hline \multirow{3}{*}{ Nazionalità } & \multicolumn{2}{|c|}{ sudanese } & \multicolumn{2}{|c|}{$44,6 \%$} & \multicolumn{2}{|c|}{$76 \%$} & \multicolumn{2}{|c|}{$39 \%$} & $17,9 \%$ & \\
\hline & \multicolumn{2}{|c|}{ sud sudanese } & \multicolumn{2}{|c|}{$36,6 \%$} & \multicolumn{2}{|c|}{$20,4 \%$} & \multicolumn{2}{|c|}{$44 \%$} & $50 \%$ & \\
\hline & \multicolumn{2}{|l|}{ altra } & \multicolumn{2}{|c|}{$17,8 \%$} & \multicolumn{2}{|c|}{$3,6 \%$} & \multicolumn{2}{|c|}{$17 \%$} & 32,1 & \\
\hline
\end{tabular}

NB. La nazionalità non è stata rilevata nel questionario. I dati si riferiscono alle informazioni dell'ufficio immatricolazioni e riflettono le percentuali sul totale degli studenti per ogni programma. 
È opportuno ricordare che la Guerra Civile in Sud Sudan, a partire da dicembre 2013, ha provocato negli anni a seguire un esodo di sud sudanesi verso il Sudan. Molti si sono iscritti al CCST, principalmente ai diplomi di IT (ITA e ITM), poiché vi trovano delle agevolazioni economiche e un ambiente che rispetta la loro diversità culturale. Questi studenti hanno completato il ciclo di scuola primaria e secondaria in inglese e hanno tra $\mathrm{i}$ 20 e i 30 anni: sono quindi leggermente più maturi rispetto agli altri che hanno fra i $18 \mathrm{e}$ 21 anni, per via del travagliato percorso di studi nel loro paese.

Anche il corso di laurea in scienze informatiche ha un'alta percentuale di studenti sud sudanesi $(36,62 \%)$, ma coloro che hanno studiato in inglese prima dell'accesso all'università sono molti meno (circa 24\%). Questo si spiega con il fatto che in buona parte sono studenti nati e cresciuti in Sudan da famiglie sud sudanesi rifugiatesi in Sudan durante la guerra che portò il Sud Sudan a dichiarare l'indipendenza nel 2011. La maggior parte di coloro che sono stati inseriti nella categoria "altri" sono eritrei, nati in Sudan. L'Eritrea è il secondo paese per numero di rifugiati in Sudan.

Il questionario proseguiva chiedendo agli studenti con che device accedessero a internet.

La maggior parte $(67 \%)$ possiede uno smartphone, che è lo strumento più comune per il collegamento (55,17\%). Solo il $41,63 \%$ possiede un computer portatile e questa percentuale è più alta tra gli studenti dei corsi di scienze informatiche (59,38\%), per i quali il $p c$ è uno strumento di studio e lavoro. Il college ha predisposto laboratori di informatica aperti a tutti, che sono diventati il principale luogo da cui connettersi, per il 22,17\% degli studenti.

Tabella 3. Accesso a Internet

\begin{tabular}{|c|c|c|c|c|c|c|c|c|c|c|c|}
\hline & & \multicolumn{2}{|c|}{$\mathrm{CS}$} & \multicolumn{2}{|c|}{ EN } & \multicolumn{2}{|c|}{ ITA } & \multicolumn{2}{|c|}{ ITM } & \multicolumn{2}{|c|}{ Totale } \\
\hline & & No. & $\%$ & No. & $\%$ & No. & $\%$ & No. & $\%$ & No. & $\%$ \\
\hline \multirow{4}{*}{ 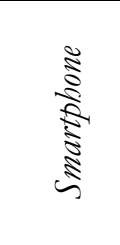 } & No & 19 & 29,69 & 61 & 32,8 & 26 & 33,77 & 23 & 29,11 & 129 & 31,77 \\
\hline & Sì & 45 & 70,31 & 125 & 67,2 & 51 & 66,23 & 51 & 64,56 & 272 & 67 \\
\hline & NR & & & & & & & 5 & & 5 & 1,23 \\
\hline & Totale & 64 & & 186 & & 77 & & 79 & & 406 & \\
\hline \multirow{4}{*}{ 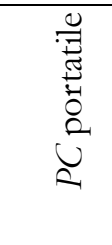 } & No & 26 & 40,63 & 116 & 62,37 & 40 & 51,95 & 49 & 62,03 & 231 & 56,90 \\
\hline & Sì & 38 & 59,38 & 69 & 37,10 & 37 & 48,05 & 25 & 31,65 & 169 & 41,63 \\
\hline & NR & & & 1 & & & & 5 & & 6 & 1,48 \\
\hline & Totale & 64 & & 186 & & 77 & & 79 & & 406 & \\
\hline \multicolumn{2}{|c|}{ Internet café } & 5 & 7,81 & 9 & 4,84 & 11 & 14,29 & 5 & 6,33 & 30 & 7,39 \\
\hline \multicolumn{2}{|c|}{$\begin{array}{l}\text { Laboratori al } \\
\text { college }\end{array}$} & 11 & 17,19 & 53 & 28,49 & 14 & 18,18 & 12 & 15,19 & 90 & 22,17 \\
\hline \multicolumn{2}{|c|}{$P C$ portatile } & 9 & 14,06 & 16 & 8,60 & 7 & 9,09 & 6 & 7,59 & 38 & 9,36 \\
\hline \multicolumn{2}{|c|}{ Smartphone } & 37 & 57,81 & 96 & 51,61 & 40 & 51,95 & 48 & 60,76 & 221 & 55,17 \\
\hline \multicolumn{2}{|l|}{ NR } & 2 & 3,12 & 12 & 6,45 & 5 & 6,49 & 8 & 1,01 & 27 & 6,65 \\
\hline \multicolumn{2}{|c|}{$\begin{array}{l}\text { Totale } \\
\text { complessivo }\end{array}$} & 64 & & 186 & & 77 & & 79 & & 406 & \\
\hline
\end{tabular}

Per valutare il tipo di utilizzo di internet, il sondaggio si è concentrato sulla posta elettronica, Whats $A p p$, la pagina Facebook del CCST e operazioni più complesse, come l'invio di allegati, nel periodo precedente all'agosto 2020. 
(C) Italiano LinguaDue 2. 2021. J. Naranjo, I. Molineri, La sfida della didattica a distanza in una Università Sudanese: la valutażone degli studenti e i risultati nei corsi di lingua italiana

Tabella 4. Utilizzo di internet

\begin{tabular}{|c|c|c|c|c|c|c|c|}
\hline & & $\mathrm{CS}$ & EN & ITA & ITM & N. Totale & Totale $\%$ \\
\hline \multirow{4}{*}{$\begin{array}{l}\text { Utente } \\
\text { e-mail }\end{array}$} & No & 20 & 68 & 33 & 26 & 147 & 36,2 \\
\hline & Sì & 43 & 111 & 41 & 45 & 240 & 59,11 \\
\hline & NR & 1 & 7 & 3 & 8 & 19 & 4,67 \\
\hline & $\begin{array}{l}\text { Totale } \\
\text { complessivo }\end{array}$ & 64 & 186 & 77 & 79 & 406 & \\
\hline \multirow{4}{*}{$\begin{array}{l}\text { Utente } \\
\text { Whats App }\end{array}$} & No & 12 & 41 & 18 & 14 & 85 & 20,94 \\
\hline & Sì & 51 & 140 & 56 & 57 & 304 & 74,88 \\
\hline & NR & 1 & 5 & 3 & 8 & 17 & 4,19 \\
\hline & $\begin{array}{l}\text { Totale } \\
\text { complessivo }\end{array}$ & 64 & 186 & 77 & 79 & 406 & \\
\hline \multirow{5}{*}{$\begin{array}{l}\text { Accesso } \\
\text { alla } \\
\text { pagina } \\
\text { Facebook } \\
\text { del CCST }\end{array}$} & Tutti i giorni & 15 & 35 & 21 & 11 & 82 & 20,20 \\
\hline & & 8 & 52 & 12 & 19 & 91 & 22,41 \\
\hline & $\begin{array}{l}\text { Una volta la } \\
\text { settimana }\end{array}$ & 40 & 94 & 37 & 43 & 214 & 52,71 \\
\hline & NR & 1 & 5 & 7 & 6 & 19 & 4,68 \\
\hline & $\begin{array}{l}\text { Totale } \\
\text { complessivo }\end{array}$ & 64 & 186 & 77 & 79 & 406 & \\
\hline \multirow{4}{*}{$\begin{array}{l}\text { Avevi mai } \\
\text { mandato } \\
\text { un } \\
\text { allegato } \\
\text { via e-mail? }\end{array}$} & No & 27 & 112 & 40 & 41 & 220 & 54,19 \\
\hline & Sì & 33 & 61 & 32 & 30 & 156 & 38,42 \\
\hline & NR & 4 & 13 & 5 & 8 & 30 & 7,39 \\
\hline & $\begin{array}{c}\text { Totale } \\
\text { complessivo }\end{array}$ & 64 & 186 & 77 & 79 & 406 & \\
\hline
\end{tabular}

\subsection{Introdurione a Moodle}

L'82,51\% degli studenti ha dichiarato di aver ricevuto le credenziali per l'accesso a Moodle al momento dell'immatricolazione, prima dell'inizio del semestre.

Durante la prima settimana (17-23 agosto), il college ha organizzato dei workshop formativi per piccoli gruppi, ma solo il 43,60\% degli studenti vi ha preso parte. La più alta percentuale si registra tra quelli di ITM $(48,10 \%)$ e ITA (48,05\%), mentre la più bassa tra gli iscritti a Lingua e Letteratura Inglese $(40,86 \%)$ che forse ne avrebbero avuto maggiore bisogno rispetto ad altri, ma all'inizio sono apparsi più ostili a questa nuova forma di apprendimento. La percentuale di partecipazione tra gli studenti di Scienze Informatiche è piuttosto bassa $(40,62 \%)$, probabilmente grazie a competenze digitali più avanzate e a un maggior livello di autonomia con gli strumenti digitali. Come già accennato, molti risiedevano fuori Khartoum e in quei giorni si registravano grossi problemi di approvvigionamento di carburante in tutto il paese, con i trasporti non garantiti. 
(C) Italiano LinguaDue 2. 2021. J. Naranjo, I. Molineri, La sfida della didattica a distanza in una Università Sudanese: la valutażone degli studenti e i risultati nei corsi di lingua italiana

Tabella 5. Valutazione dei workshop per l'utilizzo di Moodle

\begin{tabular}{lccccccccc}
\hline \multirow{2}{*}{ Valutazione } & \multicolumn{2}{c}{ CS } & \multicolumn{2}{c}{ EN } & \multicolumn{2}{c}{ ITA } & \multicolumn{2}{c}{ ITM } & \\
& No. & $\%$ & No. & $\%$ & No. & $\%$ & No. & $\%$ & Totale \\
\hline $\begin{array}{l}\text { Chiaro e } \\
\text { soddisfacente }\end{array}$ & 14 & 53,85 & 20 & 26,32 & 13 & 35,13 & 9 & 23,68 & 56 \\
$\begin{array}{l}\text { Troppo difficile } \\
\text { Mi ha aiutato }\end{array}$ & 2 & 7,69 & 13 & 17,10 & 8 & 21,62 & 15 & 39,47 & 38 \\
parzialmente & 9 & 34,62 & 38 & 50 & 14 & 37,84 & 10 & 26,32 & 71 \\
$\begin{array}{l}\text { NR } \\
\begin{array}{l}\text { Totale / \% degli } \\
\text { intervistati }\end{array}\end{array}$ & 1 & 3,85 & 5 & 6,58 & 2 & 5,41 & 4 & 10,53 & 12 \\
\hline
\end{tabular}

NB. Sono state eliminate le risposte degli studenti che non avevano partecipato ai workshop ma hanno espresso comunque un'opinione.

Dalla tabella si evince che gli studenti di Scienze Informatiche sono i più soddisfatti in merito ai workshop, tenuti dagli stessi docenti sia per i corsi di CS, ITA e ITM, diversi invece da quelli di EN. Possiamo dunque inferire che la miglior predisposizione allo studio online degli studenti di CS sia stata un fattore determinante, più che la qualità stessa dei workshop.

\subsection{Utilizzo del Learning Management System (MOODLE)}

Il college ha aperto laboratori informatici affinché gli studenti potessero accedere a Moodle da un computer e con il supporto di un assistente tecnico. L'obiettivo era quello di garantire la possibilità di iniziare le lezioni anche per coloro non in possesso di smartphone o computer. Il 46,8\% degli intervistati ha dichiarato di essere stato a conoscenza di questa opportunità, mentre il $32,26 \%$ di non averlo saputo. Più che per gli altri corsi, gli iscritti a Lingua e Letteratura Inglese erano ben informati (50\%). È importante sottolineare come i laboratori siano stati aperti vicino alle aule dove normalmente si tenevano le lezioni in presenza.

La ragione per cui molti non hanno sfruttato i laboratori è stato il costo dei trasporti pubblici $(31,53 \%)$, a causa della situazione già ampiamente descritta, oltre al fatto che molti $(15,76 \%)$ risiedevano ancora fuori Khartoum.

La qualità dei corsi disponibili sulla piattaforma è stata valutata con punteggi che vanno da 4,73/10 (ITM) a 5,37/10 (EN). È interessante notare come gli studenti di inglese, in principio più riluttanti, si siano rivelati poi più positivi di altri.

Circa la metà del numero totale di intervistati ha dichiarato di aver avuto difficoltà con Moodle, ma anche di aver acquisito maggiore fiducia nell'utilizzo del computer (49,75\%), al momento di rispondere al questionario. Al contrario invece il $26,6 \%$ ha risposto di non aver visto migliorare il proprio livello di dimestichezza dopo un mese di lavoro sulla piattaforma. Gli studenti più positivi sono quelli di scienze informatiche $(59,37 \%)$ e di inglese $(53,76 \%)$.

Il $47,54 \%$ ha affermato che lo studio a distanza ha aumentato il livello di consapevolezza, consentendo per esempio di trovare altri corsi online per l'autoapprendimento. Al contrario invece, per il 30,05\%, il LMS non è stato di alcun aiuto. Anche in questo caso, gli studenti di inglese $(52,69 \%)$ e quelli di scienze informatiche $(50 \%)$ sono stati i più positivi. 
In conclusione, i più critici sono stati gli iscritti ai diplomi di IT. In particolare, il 40,26\% degli studenti ITA ritiene che non sarà mai in grado di utilizzare il LMS, mentre solo il 18,75\% degli studenti di CS è d'accordo con una dichiarazione così radicale.

Rispetto alle difficoltà incontrate, le possibilità di scelta offerte sono indicate nella tabella 6; era possibile esprimere più di un'opzione.

Tabella 6. Principali difficoltà nell'utilizzo di Moodle

\begin{tabular}{lcccccccccc}
\hline Difficoltà & \multicolumn{1}{c}{ CS } & \multicolumn{2}{c}{ EN } & \multicolumn{2}{c}{ ITA } & \multicolumn{2}{c}{ ITM } & \multicolumn{2}{c}{ Totale } \\
& No. & $\%$ & No. & $\%$ & No. & $\%$ & No. & $\%$ & No. & $\%$ \\
\hline Dimensione: STUDENTE & & & & & & & & & & \\
$\begin{array}{l}\text { Non ho mai lavorato a lungo } \\
\text { con un } p c\end{array}$ & 19 & 29,7 & 91 & 48,9 & 30 & 39,0 & 31 & 39,2 & 171 & 42,1 \\
$\begin{array}{l}\text { Ho trovato difficile organizzare } \\
\text { da solo il mio tempo di studio }\end{array}$ & 36 & 56,3 & 98 & 52,7 & 39 & 50,6 & 39 & 49,4 & 212 & 52,2 \\
\hline
\end{tabular}

\section{Dimensione: DOCENTE}

Ho avuto difficoltà a comunicare con i docenti

$\begin{array}{llllllllll}36 & 56,3 & 81 & 43,5 & 33 & 42,9 & 36 & 45,6 & 186 & 45,8\end{array}$

\section{Dimensione: CORSO}

Alcuni corsi non erano preparati bene

$32 \quad 50,0 \quad 93 \quad 50,0 \quad 36 \quad 46,8 \quad 40 \quad 50,6 \quad 201 \quad 49,5$

\section{Dimensione: TECNOLOGIA}

Le mie credenziali di accesso a Moodle non funzionavano $\begin{array}{llllllllll}16 & 25,0 & 45 & 24,2 & 15 & 19,5 & 19 & 24,1 & 95 & 23,4\end{array}$

Non sapevo a chi rivolgermi per risolvere problemi di accesso a Moodle

$\begin{array}{llllllllll}12 & 18,8 & 52 & 28,0 & 23 & 29,9 & 26 & 32,9 & 113 & 27,8\end{array}$

La connessione Internet era lenta o discontinua

$\begin{array}{llllllllll}43 & 67,2 & 93 & 50,0 & 51 & 66,2 & 47 & 59,5 & 234 & 57,6\end{array}$

\section{Dimensione: SISTEMA}

Non sapevo dove inserire le credenziali

$\begin{array}{llllllllll}9 & 14,1 & 36 & 19,4 & 15 & 19,5 & 17 & 21,5 & 77 & 19,0\end{array}$

Ho effettuato l'accesso, ma ho trovato tutto troppo difficile

$22 \quad 34,4 \quad 86 \quad 46,2 \quad 28 \quad 36,4 \quad 32 \quad 40,5 \quad 168 \quad 41,4$

Ho effettuato l'accesso, ma mi sono perso tra tutte le informazioni

Ho avuto qualche difficoltà all'inizio, poi mi sono abituato/a

Il problema principale è stato la connessione a Internet (57,6\%), mentre il secondo la difficoltà di organizzare il proprio tempo studio $(52,2 \%)$.

Infine, gli studenti sono stati invitati ad esprimersi rispetto agli aspetti positivi e negativi dell'uso del LMS con una risposta aperta, priva di opzioni predefinite; era inoltre possibile scegliere se scrivere in inglese o arabo, per la maggioranza prima lingua. 
(C) Italiano LinguaDue 2. 2021. J. Naranjo, I. Molineri, La sfida della didattica a distanza in una Università Sudanese: la valutazione degli studenti e i risultati nei corsi di lingua italiana

Le risposte sono state raggruppate, come nella tabella precedente, per dimensione.

Tabella 7. Aspetti negativi nell'utilizzo di Moodle o aspetti che dovrebbero essere migliorati

\begin{tabular}{llc}
\hline Dimensione & Risposte & No. \\
\hline \multirow{2}{*}{ Studente } & Non sapevo usare un computer & 10 \\
& L'organizzazione del tempo a casa era difficile & 5 \\
\hline Docente & Mancanza di comunicazione e interazione con i docenti & 27 \\
\hline \multirow{5}{*}{ Corso } & Per alcune materie c'è bisogno di una spiegazione dal vivo & 26 \\
& Aggiungere video-lezioni & 24 \\
& Ho avuto problemi a consegnare compiti/assignment, specialmente & 13 \\
& attraverso il telefono, o non sapevo come farlo & \\
& Alcune lezioni non erano ben preparate & 12 \\
& Le lezioni non erano disponibili in tempo utile & 5 \\
& Aggiungere video-conferenze & 3 \\
\hline \multirow{5}{*}{ Tecnologia } & Connessione Internet & 115 \\
& Mancanza di uno smartphone & 26 \\
& Non era possibile scaricare il materiale sullo smartphone & 16 \\
& Mancanza di un computer portatile & 14 \\
& Interruzione della fornitura di energia elettrica & 9 \\
& Internet è costoso & 4 \\
\hline \multirow{2}{*}{ Sistema } & Problemi a effettuare l'accesso sulla piattaforma & 8 \\
& Non sapevo quando cominciassero le lezioni o venisse caricato il & 5 \\
\hline
\end{tabular}

NB. Le risposte con punteggio inferiore a 3 sono state eliminate

Le risposte aperte, in cui gli studenti potevano esprimersi liberamente, sono coerenti con quelle alla domanda precedente e si conferma come la difficoltà principale sia stata la connessione.

È necessario sottolineare che è stato rilevato un problema con l'applicazione Android per l'accesso diretto a Moodle, a causa del quale non era possibile scaricare i file. Gli studenti dovevano quindi accedere alla piattaforma dal sito internet del college per poter accedere al materiale caricato dai docenti. Tra gli aspetti positivi legati all'utilizzo del LMS, gli studenti hanno evidenziato i seguenti:

Tabella 8. Aspetti positivi del LMS

\begin{tabular}{llc}
\hline Dimensione & Risposte & No. \\
\hline \multirow{6}{*}{ Studente } & Ottimizzare l'uso del tempo & 40 \\
& Sfida a non temere lo studio a distanza e l'utilizzo del pc e della & 34 \\
& tecnologia / Miglioramento delle competenze digitali & \\
& Posso studiare a casa o dove preferisco e organizzarmi & 20 \\
& Incoraggiamento a fare ricerche su internet & 5 \\
& Scoperta di nuovi metodi di insegnamento e apprendimento & 4 \\
& Ora so utilizzare le e-mail & 3 \\
\hline
\end{tabular}


(C) Italiano LinguaDue 2. 2021. J. Naranjo, I. Molineri, La sfida della didattica a distanza in una Università Sudanese: la valutazione degli studenti e i risultati nei corsi di lingua italiana

\begin{tabular}{llc}
\hline \multirow{2}{*}{ Corso } & Semplice accesso alle lezioni e alle informazioni & 23 \\
& Lezioni ben organizzate & 5 \\
& Buoni contenuti & 3 \\
& Mi ha aiutato a rispettare le scadenze di consegna di compiti & 3 \\
\hline \multirow{2}{*}{ Sistema } & Facile da usare & 19 \\
& Il LMS non ha alcun aspetto positivo & 19 \\
\hline \multirow{2}{*}{ Ambiente } & Riduzione delle spese per il trasporto e relativi problemi & 52 \\
& Protezione dal COVID-19, rispetto del distanziamento sociale & 5 \\
\hline
\end{tabular}

\subsection{Risultati degli studenti di lingua italiana}

Durante le prime settimane, la comunicazione tra il docente e gli studenti è risultata molto complessa. Pochissimi prendevano parte alle sessioni di chat sincrona e quasi nessuno rispondeva ai messaggi. Monitorando le connessioni, si vedevano dei tentativi, ma l'interazione era inesistente. La situazione è migliorata in maniera significativa con il rientro degli studenti in classe, per le video conferenze con il docente in remoto e le esercitazioni con l'assistente. Durante le videoconferenze si è fatto riferimento al materiale disponibile su Moodle e si sono ripercorsi i passi per accedere alle varie attività, utilizzate anche come input didattico.

Il voto finale, come già descritto, è composto da due test parziali in itinere, valutazione continua ed esame finale. Confrontando i voti degli studenti nel primo semestre (Italiano 1 e 3) con quelli degli stessi studenti nel secondo semestre (Italiano 2 e 4), otteniamo i seguenti risultati:

Tabella 9. Media semestrale nei corsi di italiano

\begin{tabular}{|c|c|c|c|c|}
\hline & \multicolumn{2}{|c|}{$\begin{array}{l}\text { Studenti del secondo anno } \\
\qquad(\text { tot }=29)\end{array}$} & \multicolumn{2}{|c|}{$\begin{array}{l}\text { Studenti del terzo anno } \\
\quad(\text { tot }=22)\end{array}$} \\
\hline & Italiano 1 & Italiano 2 & Italiano 3 & Italiano 4 \\
\hline Media & 2,586 & 2,421 & 2,959 & 2,450 \\
\hline Varianza & 0,762 & 0,739 & 1,270 & 0,765 \\
\hline Deviazione standard & 0,873 & 0,860 & 1,127 & 0,875 \\
\hline Minimo & 1,0 & 0,8 & 0,6 & 1,2 \\
\hline Massimo & 4,1 & 4,1 & 4,7 & 4,6 \\
\hline Indice di asimmetria & $-0,267$ & $-0,80$ & $-0,719$ & 0,533 \\
\hline Curtosi & $-0,650$ & $-0,552$ & $-0,122$ & $-0,30$ \\
\hline Shapiro-Wilk (p) & 0,183 & 0,811 & 0,077 & 0,665 \\
\hline $\begin{array}{l}\text { Indice di correlazione } \\
\text { appaiato }\end{array}$ & \multicolumn{2}{|c|}{0,722} & \multicolumn{2}{|c|}{0,863} \\
\hline Differenze appaiate & \multicolumn{2}{|c|}{$\mathrm{t}(28)=1,380$} & \multicolumn{2}{|c|}{$\mathrm{t}(21)=2,952$} \\
\hline $\mathrm{p}$ (due code) & \multicolumn{2}{|c|}{0,179} & \multicolumn{2}{|c|}{0,008} \\
\hline
\end{tabular}


Poiché il valore $\mathrm{p}>0.05$ per il test di Shapiro-Wilk in tutti i casi, possiamo concludere che i dati derivano da una distribuzione normale e quindi i test $\mathrm{t}$ possono essere eseguiti su entrambi i campioni.

In entrambi i casi la media nel secondo semestre è inferiore rispetto al primo. Il test $\mathrm{t}$, poiché $\mathrm{p}>0.05$, indica che la differenza appaiata tra la media di "Italiano 1" e quella di "Italiano 2" non è statisticamente rilevante.

Per quanto riguarda invece la differenza tra "Italiano 3" e "Italiano 4", il valore p $<0.05$, e dunque è statisticamente rilevante.

Come mostra la tabella, l'asimmetria è negativa per "Italiano 1,2 e 3", cioè i voti si concentrano verso i valori più alti, mentre per "Italiano 4" l'asimmetria è positiva, con voti dunque concentrati verso i valori più bassi.

La curtosi negativa mostra come la distribuzione sia platicurtica (iponormale), con i voti degli studenti distribuiti nel range tra il minimo e il massimo, invece che essere concentrati intorno ai valori della media.

\section{DisCussione}

\subsection{Dimensione studente}

Il 42,1\% degli intervistati ha dichiarato di non aver mai lavorato molto con un computer prima di questo corso (Tabella 6 ). La mancanza di familiarità con lo strumento informatico, particolarmente per gli studenti della laurea in lingua inglese, di solito genera ansia, che ha un impatto negativo sul livello di soddisfazione dell'apprendente online (Sun et al., 2008). Alcuni partecipanti hanno infatti indicato la scarsa conoscenza dell'uso del $p c$ tra gli aspetti negativi dell'utilizzo di Moodle (Tabella 7). D'altro canto però, 34 studenti hanno affermato che il mese di studio su LMS ha costituito una sfida che ha aiutato a non temere lo studio online e l'uso del computer, e che in generale le loro competenze informatiche sono migliorate (Tabella 8).

Un altro aspetto rilevante della ricerca è che il 52,2\% degli intervistati ha sostenuto di aver avuto difficoltà a organizzare il proprio tempo (Tabella 6). Ciò ha probabilmente a che fare con la tradizione scolastica sudanese e il contesto, in cui la maggior parte degli studenti non ha uno spazio personale da dedicare allo studio a casa e le scuole non dispongono di biblioteche o aule studio. Per questo motivo, il processo di apprendimento è confinato alle attività svolte in classe, con una metodologia basata sulla memorizzazione.

Questo aspetto risulta determinante poiché diversi studi mostrano come «la maggior parte degli studenti con la media più alta ( 3.5 su 5 e oltre) possiede forti capacità di gestione del tempo» (Miertschin, Goodson, Stewart, 2015: 26). Altre ricerche mostrano però come uno dei benefici delle piattaforme per lo studio a distanza sia il miglioramento della capacità di autogestione (Bhuasiri, Xaymoungkhoun, Hangjung, Jae, 2012; Miertschin et al., 2015). 40 studenti del CCST hanno rilevato come invece proprio l'uso della piattaforma li abbia aiutati a massimizzare il proprio tempo e 20 studenti trovano che la possibilità di autogestione e studio da casa o da dove si voglia costituisca un aspetto positivo (Tabella 8).

\subsection{Dimensione docente}

L'aspetto più critico riferito a questa dimensione è la difficoltà che gli studenti hanno sperimentato nell'interazione con gli insegnanti (Tabelle 6 e 7). È inoltre interessante 
sottolineare come questo fenomeno sia più rilevante per gli iscritti ai corsi di Scienze informatiche $(56,3 \%)$.

Interazione e partecipazione sono fondamentali per un'eccellente esperienza di apprendimento in rete (Salomon, 2000). Infatti, l'obiettivo del LMS è «creare comunità online, non solo erogare contenuti e organizzare la documentazione dei corsi» (Crosslin, 2006 , p. 505). Inoltre, «la rapida risposta del docente influenza in modo significativo il livello di soddisfazione degli apprendenti» (Sun et al., 2008: 1186; citando Arbaugh, 2002; Thurmond et al., 2002), anche se gli autori si concentrano sull'atteggiamento del docente verso l'e-learning, aspetto di cui invece non si occupa questa ricerca.

Alcuni docenti, di fronte alle difficoltà di interagire con gli studenti, hanno creato gruppi Whats $A p p$ invece di sfruttare gli strumenti di Moodle, quali la chat o la posta elettronica. Anche il diario dell'insegnante di italiano riporta questo problema, dalla sua prospettiva.

\subsection{Dimensione corso}

Secondo il 49,5\% degli intervistati, i corsi disponibili sulla piattaforma non erano ben preparati e non si evidenziano differenze significative tra i diversi programmi accademici (Tabella 6). Tra gli aspetti che dovrebbero essere migliorati, 26 studenti hanno manifestato il bisogno di spiegazioni dal vivo e di disporre di un maggior numero di video lezioni (Tabella 7).

Diversi studi hanno infatti dimostrato come la qualità e flessibilità dei corsi abbia un impatto significativo sul livello di soddisfazione dell'apprendente (Sun et al., 2008) e che «una buona programmazione di corsi, curriculum e materiali didattici faciliti l'esperienza formativa» (Bhuasiri, 2012: 847; citando Brophy, 2000).

D'altro canto, 23 studenti hanno apprezzato la facilità di accesso al docente e alle informazioni (Tabella 8).

In relazione alla dimensione corso, è necessario sottolineare che gli studenti hanno valutato la qualità dei corsi caricati sul LMS con punteggi compresi tra 4,73/10 e 5,37/10, che indicano un livello di soddisfazione generalmente basso.

\subsection{Dimensione tecnologia}

In riferimento alla tecnologia e alle infrastrutture, la più grande difficoltà rilevata dal $57,5 \%$ degli studenti è stata la connessione a Internet (Tabella 6). Ciò emerge con forza come uno degli aspetti negativi nell'utilizzo di Moodle (Tabella 7), rilevato da 115 partecipanti. La cornice teorica di questa ricerca ha già descritto i problemi di accesso alla rete in Sudan a cui si aggiungevano, è opportuno ribadirlo, quotidiane interruzioni di corrente in quel periodo.

Lo studio di Sun et al. conclude che «nessun fattore afferente alla dimensione tecnologica ha un effetto significativo sul livello di soddisfazione degli e-learnen) (2008: 1195). Bisogna tuttavia considerare che la ricerca di Sun et al. è stata condotta a Taiwan, dove la connessione Internet è certamente migliore di quella sudanese. Senza ombra di dubbio, avere difficoltà nell'accesso a Internet per cause esterne scoraggia docenti e studenti, oltre ad avere un impatto negativo sull'ambiente formativo. 


\subsection{Dimensione sistema}

Il 41,4\% dei partecipanti ha dichiarato di aver trovato Moodle troppo complesso una volta entrati, mentre per il 41,6\% è stato difficile all'inizio, ma poi è subentrata maggiore consapevolezza (Tabella 6).

Nelle risposte aperte raccolte nella Tabella 8, 16 studenti rispettivamente hanno affermato di aver trovato Moodle molto facile da usare o completamente inutile.

La piattaforma incoraggia gli studenti con competenze digitali e li aiuta ad adattarsi alla società dell'informazione e della conoscenza, come anche a continuare il percorso di educazione permanente una volta completati gli studi (Sun et al., 2008). Alcuni hanno percepito questi benefici, poiché hanno dichiarato che l'utilizzo del LMS ha permesso loro di scoprire nuovi modi di insegnare, imparare ed effettuare ricerche su internet (Tabella $8)$.

\subsection{Dimensione ambiente}

52 studenti hanno indicato tra gli aspetti positivi dell'utilizzo di Moodle la riduzione delle difficoltà e delle spese di trasporto (Tabella (8). Questa risposta è senz'altro frutto della situazione contingente che viveva il paese.

Tuttavia, normalmente questa dimensione si riferisce all'ambiente di apprendimento e questa ricerca non si è addentrata nelle dinamiche di interazione attraverso Moodle, aspetto fondamentale per la valutazione.

\section{CONCLUSIONI, LIMITI E RACCOMANDAZIONI}

\subsection{Limiti}

Uno dei limiti di questo studio è la mancanza di dati sulle competenze digitali dei docenti prima dell'introduzione della didattica a distanza, come sulle specifiche abilità relative allinsegnamento da remoto e sulla percezione che $\mathrm{i}$ docenti hanno di questa modalità, che, secondo Sun et al. (2008), può avere un impatto determinante sul livello di soddisfazione degli apprendenti.

Come mostra la Tabella 2, per la maggior parte degli studenti del CCST, e in particolare quelli dei programmi di laurea, l'arabo è la lingua di studio nella scuola primaria e secondaria. La mancanza di dati riguardo il livello di competenza linguistica in inglese, anche in riferimento al personale, per esempio attraverso gli esami IELTS, permette di avere solo una prospettiva parziale rispetto a possibili difficoltà nell'utilizzo del LMS in inglese. Per gli iscritti della Laurea in Lingua e Letteratura Inglese, questa ipotetica difficoltà potrebbe acuirsi con l'introduzione di una terza lingua, italiano o spagnolo, come previsto dal piano di studi.

\subsection{Conclusioni e raccomandazioni}

Gli studenti del CCST utilizzano Internet più della media nazionale sudanese (31\%); come mostra la Tabella 3, solo il 9,36\% dispone di un computer portatile, mentre il 55,17\% accede alla rete attraverso lo smartphone, che certamente non è lo strumento di studio ideale, sia per quel che riguarda sia la lettura che la videoscrittura. 
Questo studio rimarca l'importanza dei corsi di alfabetizzazione informatica nel primo semestre dei programmi di laurea, in particolare nei corsi di Laurea in Lingua e Letteratura Inglese, al fine di migliorare le competenze digitali. Certamente il problema sarebbe assai più determinante se ci si trovasse nelle circostanze di dover iniziare il primo semestre interamente online, cosa che, in questo caso, non è avvenuta.

Questa ricerca mostra anche come sia indispensabile fornire agli studenti strumenti per la gestione del tempo e dello studio individuale, soprattutto in un contesto come quello sudanese in cui la formazione scolastica è ancora profondamente legata alla memorizzazione.

Dalla ricerca emerge l'esigenza di test d'ingresso per la lingua inglese all'inizio del percorso accademico, senza necessariamente imporre un requisito minimo per l'accesso, ma predisponendo poi corsi accessibili tutto l'anno per raggiungere almeno punteggi compresi tra 6.5 e 8, corrispondenti ai livelli B2 e C1 del Quadro Comune Europeo di riferimento delle Lingue, propedeutici alla laurea. Per quanto attiene alla Laurea in Lingua e Letteratura Inglese, la certificazione linguistica obbligatoria sia in inglese (B2/C1) che nella terza lingua di studio - italiano o spagnolo (A2, come previsto dal sillabo) costituirebbe forse un elemento di maggiore chiarezza rispetto al carico lavorativo da affrontare, favorendo così un atteggiamento più proattivo. Per quanto riguarda il corpo docente, la certificazione IELTS aggiungerebbe un traguardo misurabile ai percorsi di aggiornamento professionale.

Per promuovere più alti standard di qualità, trasparenza e consapevolezza, fondamentale sia per comprendere come raggiungere gli obiettivi, ma anche ai fini della formazione continua degli insegnanti, documenti di carattere generale, quali guide dello studente e griglie di valutazione, con chiare specifiche degli obiettivi formativi, carico di lavoro e tempi di studio, dovrebbero essere standardizzati e condivisi. Questo processo di formalizzazione e standardizzazione dell'offerta formativa è in atto da tempo al CCST, con risultati differenti a seconda dei dipartimenti. Visto anche l'intensificarsi dell'attività di internazionalizzazione e scambio accademico, è di fondamentale importanza preparare studenti e docenti a interagire con un sistema universitario, come per esempio quello europeo, che fa dell'autonomia un punto di forza.

Questa ricerca ha anche evidenziato un problema di comunicazione, certamente legato anche alle competenze digitali di tutti gli attori coinvolti e all'uso limitato al quotidiano che fanno degli strumenti informatici. Nonostante la maggioranza degli studenti abbia un account e-mail attivo, come mostra la Tabella 4, si rileva una forte riluttanza a utilizzarlo regolarmente nelle comunicazioni ufficiali, anche attraverso Moodle. Si tende invece a preferire la creazione di gruppi Whats $A p p$, in cui non solo richiamare comunicazioni importanti, ma condividere file, compiti, spiegazioni, dubbi e domande. Ciò si spiega certamente con il fatto che è proprio lo smartphone il principale strumento di studio; la comunicazione via WhatsApp risente però delle semplificazioni dettate da tempi, linguaggio e registro tipici dei servizi di messaggistica, lasciando da parte la necessità di tempo e cura nell'impostare il testo di un'e-mail formale. Sempre nell'ottica dei programmi di scambio con l'estero, o della ricerca attiva di un lavoro, di altri corsi o borse di studio, il corretto uso delle e-mail è senz'altro una competenza da addestrare, a cui si può provvedere dotando tutti di indirizzo ufficiale del college, sul quale far confluire tutte le comunicazioni e da sincronizzare con il servizio di posta interno a Moodle.

Anche i docenti, infatti, sono sembrati più inclini a utilizzare Whats $A p p$ come mezzo di comunicazione più efficace, perché più immediato. La raccolta dati e la valutazione delle competenze digitali degli insegnanti permetterebbe di strutturare strategie condivise che mirino a metterli nelle condizioni di produrre e gestire al meglio il materiale per i corsi online, prestando molta a attenzione ai sistemi di monitoraggio da remoto dell'attività degli 
studenti, fornendo supporto e guida personalizzata attraverso sistemi sincroni e asincroni. Queste abilità aggiungerebbero valore anche in caso di corsi solo in presenza o ibridi.

Durante quel particolare semestre di attività online e ibrida, diversi professori sono ricorsi all'uso dei social media anche per lezioni in diretta. Compatibilmente con i limiti imposti dalle caratteristiche tecniche della connessione Internet in Sudan, i social media offrono senz'altro una piattaforma di più immediata comprensione da parte degli studenti, come specificato al punto 2.2, e permettono poi di fornire supporto alla circolazione delle informazioni legate a calendari, eventi, scadenze; insieme a una sezione news sulla pagina del sito di ateneo, potrebbero essere uno strumento valido per venire incontro a coloro che, come indicato nella sezione 4.3, non sono venuti a conoscenza di alcune delle opportunità formative messe a disposizione nel periodo di chiusura.

In conclusione, i risultati di questo studio indicherebbero che corsi online progettati con cura, ricchi di risorse di facile accesso, metodi integrati di erogazione delle lezioni e della valutazione, sommativa e continua, garantiscono aderenza agli obiettivi formativi, come mostra l'analisi dei dati sui corsi di italiano.

Risulta anche evidente che gli studenti comprendono il potenziale dell'educazione online e che attenzione specifica deve essere prestata allo sviluppo delle competenze trasversali, per permettere loro di sfruttare appieno le potenzialità del LMS, al netto delle difficoltà di connessione o delle interruzioni di fornitura elettrica. Conclusioni simili si possono trarre per i docenti e i membri dello staff. Attraverso una dettagliata analisi del loro retroterra linguistico e digitale e dei loro punti di forza e delle criticità, è possibile disegnare un processo di armonizzazione formazione continua di qualità che realizzi con successo la transizione verso l'erogazione di corsi integrati.

\section{RIFERIMENTI BIBLIOGRAFICI}

Ahmed Albasheer Osman N. (2014), The acceptance and use of information and communication technologies by staff members in Khartoum state's universities (Sudan). [Tesi di dottorato, Faculty of Education. Dresden University of Technology].

Bhuasiri W., Xaymoungkhoun O., Hangjung Z., Jae J. R. (2012), "Critical Success Factors for E-Learning in developing Countries: A Comparative Analysis Between ICT Experts and Faculty", in Computers \& Education, 58, 2, pp. 843-855: https://doi.org/10.1016/j.compedu.2011.10.010.

Cavus N., Zabaldi T. (2014), "A Comparison of Open Source Learning Management Systems", in Procedia - Social and Behavioral Sciences, 143, pp. 521-526: https://doi.org/10.1016/J.SBSPRO.2014.07.430

Crosslin M. (2009), "Course Management Meets Social Networking in Moodle", in Rogers P. et alii (eds.), Encyclopedia of Distance Learning, Information Science Reference, pp. 505-509: https://doi.org/10.4018/978-1-60566-198-8.

Graf S., List B. (2005), "An evaluation of open-source e-learning platforms stressing adaptation issues", in Fifth IEEE International Conference on Advanced Learning Technologies (ICALT'05): https:/ / doi.org/10.1109/ICALT.2005.54

Hamdy A. (2007), ICT in Education in Sudan, World Bank, Washington DC: https://openknowledge.worldbank.org/bitstream/handle/10986/10660/463630 BRI0Box31an010ICTed0Survey111.pdf? sequence $=1$ \&isAllowed $=\mathrm{y}$.

Hananto A. (2019), Rank of Countries with Fastest (and Slowest) Internet in The World 2019: https://seasia.co/2019/07/12/rank-of-countries-with-fastest-and-slowestinternet-in-the-world-2019. 
(C) Italiano LinguaDue 2. 2021. J. Naranjo, I. Molineri, La sfida della didattica a distanza in una Università Sudanese: la valutażone degli studenti e i risultati nei corsi di lingua italiana

Hassan Abdelrahman O. (2010), "Online Education in a Developing Country: The Case of Sudan", in 2nd International Symposium on Information Management in a Changing World, Hacettepe University, Ankara, pp. 306-308: https://doi.org/10.13140/2.1.3050.6887.

Internet World Stats. (2020), Internet Users Statistics for Africa: https://www.internetworldstats.com/stats1.htm.

Kemp S. (2020), Digital 2020: Sudan: https://datareportal.com/reports/digital-2020-sudan.

Miertschin S. L., Goodson C. E., Stewart B. L. (2015), "Time Management Skills and Student Performance in Online Courses", in 122nd ASEE Annual Conference and Exposition, June 14-17, 2015, American Society for Engineering Education, Seattle: https://peer.asee.org/time-management-skills-and-student-performance-inonline-courses.

North B., Goodier T., Piccardo E. (2018), Common European Framework of Reference for Languages. Learning, teaching, assessment. Companion Volume with new descriptors, Council of Europe: https://rm.coe.int/cefr-companion-volume-with-new-descriptors2018/1680787989.

Sawahel W. (2020), "Universities turn to online learning as pandemic continues", in University World News, African Edition: https://www.universityworldnews.com/post.php?story=20200723072151443.

Sun P. C., Tsai R. J., Finger G., Chen Y. Y., Yeh, D. (2008), "What drives a successful eLearning? An empirical investigation of the critical factors influencing learner satisfaction", in Computers \& Education, 50, 4, pp. 1183-1202.

The Economist (2020), The Inclusive Internet Index 2020. Intelligence Unit: https://theinclusiveinternet.eiu.com/explore/countries/LB/performance/indicat ors/.

Yin R. K. $\left(2002^{2}\right)$, Case Study Research. Design and Methods, SAGE, Thousand Oaks.

\section{Sitografia}

Moodle (2020), Registered Moodle Sites: https://stats.moodle.org/sites/. 